\section{The cerebellum in eye movement control: nystagmus, coordinate frames and disconjugacy}

Eye (2015) 29, 299; doi:10.1038/eye.2014.306

Correction to: Eye (2015) 29, 191-195; doi:10.1038/ eye.2014.271; published online 14 November 2014

Since the online publication of the above article, the authors have noticed an error. In the last sentence of the second to last paragraph on page 2 of the manuscript, 'that' had been incorrectly changed to 'when'. The sentence has now been changed to:
VR Patel and DS Zee

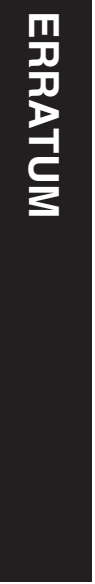

We will invoke an analogous pathogenesis for certain abnormalities of static alignment related to the incorrect assumption that the head is tilted (see below).

The Publisher sincerely apologises for the inconvenience caused.

This error has now been rectified, and the corrected article appears in this issue. The html and online pdf versions have also been rectified, and now carry the corrected paper. 